Degradation of image quality induced by thermal loads and slope errors in an XUV high resolution synchrotron radiation monochromator

This article has been downloaded from IOPscience. Please scroll down to see the full text article.

1994 Pure Appl. Opt. 3323

(http://iopscience.iop.org/0963-9659/3/3/016)

View the table of contents for this issue, or go to the journal homepage for more

Download details:

IP Address: 159.149.45.88

The article was downloaded on 11/07/2012 at 15:38

Please note that terms and conditions apply. 


\title{
Degradation of image quality induced by thermal loads and slope errors in an xuv high resolution synchrotron radiation monochromator
}

\author{
C Lenardi \\ † Consorzio INFM, Unitá Politecrico di Milano, Dipartimento di Fisica, piazza \\ Leonardo da Vinci 32, I-20133, Milano, Italy \\ $\ddagger$ Dipartimento di Elettronica, via Abbiategrasso 209, I-27100 Pavia, Italy
}

Received 13 September 1993, in final form 26 November 1993

\begin{abstract}
The limiting effects to the performances of an optical device, such as an XUV monochromator inserted in a synchrotron radiation facility, are mainly manufacturing slope errors and thermal loads induced by the optical beam itself. We have performed numerical simulations on such effects in low energy section of a high resolution, wide spectral range monochromator for the storage ring ELETTRA in Trieste. The instrument will be used in the dedicated surface physics beamline ALOISA (advanced line for overlayer interface and surface analysis). Thermal effects have been evaluated by using finte element antalysis. Thermal induced deformations and slope errors have been included in a ray tracing program. The resulting aberrations at the output of the instrument have been evaluated for the extreme conditions of maximum power density and asymmetrical illumination of the second mirror. The results stress the relevance of manufacturing slope errors in limiting spatial and spectral resolution.
\end{abstract}

\section{Introduction}

A high resolution monochromator covering a wide spectral range has been proposed for the ALOISA beamline at the ELETTRA storage ring $[1,2]$. The system has a fixed exit slit and output beam direction. It is based on a four optical elements geometry in which the two extreme off-axis paraboloids (mirrors 1 and 3) act as collimator and imager, respectively, as illustrated in figure 1 . The dispersion system operates then in parallel light and consists of a plane mirror (mirror 2) plus an interchangeable plane grating for the lower energy range. It can be exchanged with a channel cut crystal dispersing unit for operation in the high energy region.

The double Bragg deflection requires that input and output beams are parallel. The same geometry is then imposed upon the low energy dispersing system by requiring that the deflection produced by the grating exactly compensates the one given by the plane mirror. Due to the extension of the spectral range covered between $250 \mathrm{eV}$ to $8 \mathrm{keV}$, the angles of incidence on the parabolic mirrors are extremely high, $89.5^{\circ}$. This layout is geometrically aberration free in the whole spectral range for an on-axis point source. Aberrations originating from errors in the figuring of the optical surfaces are minimized by using the so-called sagittal focusing geometry, in which the plane of dispersion is parallel to the sagittal direction for the paraboloids. The instrument is designed to operate with an extended source. Scanning of the wavelength is achieved 


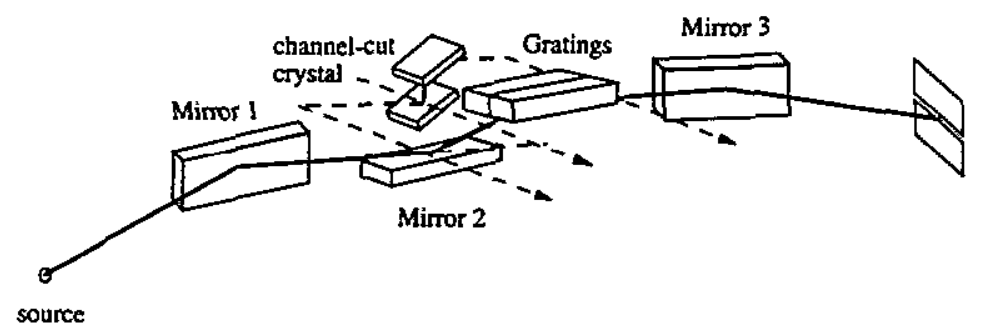

Figure 1. Optical layout of the monochromator. Mirror 1, 2, parabolic mirrors; mirror 3, plane mirror; $\mathrm{G}$, grating; $\mathrm{C} 1, \mathrm{C} 2$, silicon crystals.

in the low energy section by independent rotations of the grating and the plane mirror. Both elements rotate around axes parallel to the grating grooves. While the grating rotates around an axis centred on its optical surface, the axis of the rotation of the mirror lies outside the reflecting surface.

The optical design of the low-energy ALOISA monochromator has shown that a well focused beam $\left(30 \times 30 \mu \mathrm{m}^{2}\right)$ with a high degree of monochromaticity $\left(\Delta \lambda / \lambda \approx 2 \times 10^{-4}\right)$ may be delivered to the experimental chamber in the range between $250 \mathrm{eV}$ to $2 \mathrm{keV}$. Several factors may degrade these performances. Mechanical problems such as misalignments, external vibrations, thermal gradients and related structural deformations have to be dealt with. Vacuum contamination may affect the spectral reflectivity of the optical system. Furthermore, heating of optical elements induced by the absorbed radiation results in surface deformations affecting the optical properties of the system. This effect is coupled with the intrinsic figure and roughness errors of manufactured mirrors. All this results in a broadened focal spot of the beam on the experimental chamber with degraded spectral resolution. Reducing these detrimental effects is a central problem in synchrotron radiation technology, since they partially may offset any advantage deriving from third generation high brilliance machines such as ELETTRA.

In the present paper we have investigated the effects on the optical performances induced by thermal loads and slope errors for the ALOrSA beamline. This study refers to the lower energy part of the monochromator, i.e. the one using a plane grating as a dispersing element. Optical aberrations induced by these two effects are studied simultaneously by means of ray tracing techniques.

\section{Source characterization}

We have extensively studied the characteristics of the light source for the AloISA beamline in order to apply realistic heat loads on each optical element. Following the ALOrSA insertion device (XD) specifications $[3,4]$ and the optical instrument design [1], we have evaluated the absorbed power along the beamline to calculate the thermal field and the induced deformations on the optical surfaces. A pinhole is positioned in front of the beamline in order to reduce the thermal loads on the first optical surfaces and to select an appropriate energy range.

The power density distribution of each harmonic from the source has been evaluated using the code URGENT [5], which takes into account the non-zero emittance of the electron beam and finite number of undulator periods. From these data the spectral incident flux on each optical element has been evaluated. The absorbed fractions of the 
Table 1. Parameters of the ELETTRA machine and Alois a beamline. (a) ELETTRA parameters [12]. (b) ALOISA insertion device parameters [3, 4]. (c) ALOISA layout parameters.

\begin{tabular}{lll} 
(a) & & \\
Electron beam energy & 2.0 & $\mathrm{GeV}$ \\
Maximum circulating current at $2.0 \mathrm{GeV}$ & 400 & $\mathrm{~mA}$ \\
Natural emittance $\varepsilon$ & $7.1 \times 10^{-9}$ & $\pi \mathrm{m} \mathrm{rad}$ \\
Beam size in the ID: horizontal $\sigma_{x}^{\mathrm{c}}$ & 240 & $\mu \mathrm{m}$ \\
Beam size in the ID: vertical $\sigma_{y}^{\mathrm{c}}$ & 43 & $\mu \mathrm{m}$ \\
Beam divergence in the ID: horizontal $\sigma_{x}^{\prime c}$ & 29.0 & $\mu \mathrm{rad}$ \\
Beam divergence in the ID: vertical $\sigma_{y}^{\prime e}$ & 16.5 & $\mu \mathrm{rad}$ \\
& & \\
(b) & 73 & $\mathrm{~mm}$ \\
Period length & 20 & $\mathrm{~mm}$ \\
Minimum gap & 21 & \\
Number of periods per section & 1 & \\
Number of sections & & \\
(c) & & $\mathrm{mrad}$ \\
Pinhole aperture: horizontal $\sigma_{x}^{\prime}$ & 0.166 & $\mathrm{mrad}$ \\
Pinhole aperture: vertical $\sigma_{y}^{\prime}$ & 0.231 & $\mathrm{~m}$ \\
Source-first mirror distance & 21 & \\
\hline
\end{tabular}

t In the future the number of sections will probably increase up to three.

impinging beam have been calculated from the spectral reflectances [6] of the optical surfaces. Table 1 summarizes the parameters needed to identify the working conditions of the source, including the performance parameters of the ELETTRA storage ring and the sizes of the pinhole. A single undulator section has been considered in the calculations. The beam selector is tailored according to the low energy range, where the angular spread is largest; smaller pinholes might be used in the high energy range, but in the following calculations we have always assumed the same low energy aperture which in any case corresponds to the worst conditions.

The following simulations have been performed assuming maximum electron beam energy of $2 \mathrm{GeV}$. They refer to worst case conditions and the results provide an upper limit to the degradation of the performances of the monochromator.

\section{Thermal loads and finite element method analysis}

It is apparent that the thermal load on mirror 1 (see figure 1) is not relevant due to the extreme grazing angle of the impinging radiation. On mirror 2 , instead, the angle of incidence is smaller and variable. Thus, the thermal load may be substantial. Many configurations have been evaluated and two extreme cases occur. A first one corresponds to maximum power density on the second mirror at a transmitted photon beam at $250 \mathrm{eV}$. A second case occurs at maximum absorbed power on the same mirror at $2 \mathrm{keV}$.

Table 2 summarizes the configuration of the monochromator in the two cases considered. In the evaluation of the thermal loads the incidence angles of the beam on the plane mirror and on the grating are the same. This corresponds to using the diffraction optics at zero order, i.e. in the most critical situation (being diffraction angles at order used always smaller than the incidence angles). 
Table 2. Monochromator configurations for different values of the deflection parameter $K$ and total absorbed power and power density for each optical element.

\begin{tabular}{llllll}
\hline Element & $\begin{array}{l}\text { Work } \\
\text { energy } \\
(\mathrm{keV})\end{array}$ & $K$ & $\begin{array}{l}\text { Incident } \\
\text { angle } \\
(\mathrm{deg})\end{array}$ & $\begin{array}{l}\text { Absorbed } \\
\text { power } \\
(\mathrm{W})\end{array}$ & $\begin{array}{l}\mathrm{d} P / \mathrm{d} A \\
\left(\mathrm{~W} \mathrm{~mm} \mathrm{~m}^{-2}\right)\end{array}$ \\
\hline Mirror 1 & 0.25 & 1.47 & 89.50 & 1.68 & $1.1 \mathrm{e}-3$ \\
& 2 & 4.90 & & 5.31 & $3.5 \mathrm{e}-03$ \\
Mirror 2 & 0.25 & 1.47 & 80.38 & 11.48 & $1.4 \mathrm{e}-1$ \\
& 2 & 4.90 & 88.25 & 14.27 & $3.3 \mathrm{e}-02$ \\
Grating & 0.25 & 1.47 & 80.38 & 0.28 & $3.5 \mathrm{e}-3$ \\
& 2 & 4.90 & 88.25 & 3.08 & $7.2 \mathrm{e}-3$ \\
Mirror 3 & 0.25 & 1.47 & 89.50 & $2.0 \mathrm{e}-3$ & $1.3 \mathrm{e}-6$ \\
& 2 & 4.90 & & 0.45 & $2.9 \mathrm{e}-4$ \\
\hline
\end{tabular}

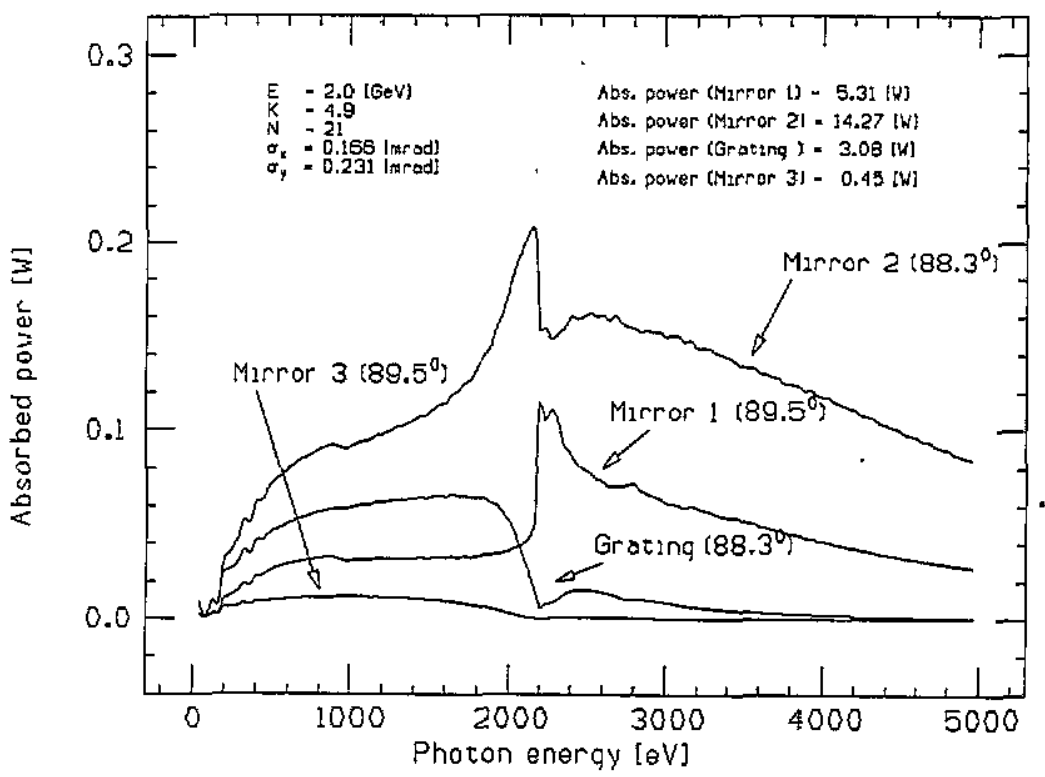

Figure 2. Absorbed power (W) by each optical element plotted against incident photon energy at the electron beam energy of $2.0 \mathrm{GeV}$ and $K=4.9$.

Figure 2 illustrates the absorbed power at the four optical elements for each impinging harmonic versus the corresponding peak energy. The grazing angles and the total absorbed powers of each optical element are also indicated and refer to the configuration at $2 \mathrm{keV}$ of table 1 .

Thermally induced deformations have been evaluated for the two critical cases described above. The analysis has been carried out by using the finite element code ABAQUS [7]. 
Deformations have been evaluated on the two first mirrors only, since the absorbed beam power by the optical elements downstream is practically negligible. For the first parabolic mirror the beam spot size spreads over its whole length and the axis of the beam hits the centre of the mirror, resulting in a four-fold symmetry. Only a quarter of the mirror itself needs then to be considered. Two-dimensional thermal-stress analysis has been performed on sagittal and tangential principal half-sections of the element. This proves a more accurate model in the meridional direction, than in sagittal one.

Unfortunately, similar symmetry considerations cannot be used for mirror 2 . In fact, by scanning the photon energy in the range between $250 \mathrm{eV}$ to $2 \mathrm{keV}$, the illuminated area 'runs' on the plane mirror along the longitudinal direction. Moreover, when the two gratings are exchanged, the illuminated area shifts from one side of the optical surface to the other. Therefore, in the two-dimensional model, several sagittal and tangential complete mirror cross sections need to be considered in order to evaluate thermal deformations accurately.

In table 3, the physical constants of the considered blanks are reported. Moreover, the dimension of the optical elements and the spot sizes in each analysed configuration are collected in table 4 . In the structural analysis the kinematic conditions constrain the rear of the blank of each optical element on its support.

For the first mirror we have considered several different positions of the heat exchanger. However, the slope of the irradiated area is largely independent of the

Table 3. Physical constants of the two first optical elements.

\begin{tabular}{lll}
\hline Parameter & Value & Units \\
\hline Blank material & $\mathrm{SiC}$ & \\
Coating material & $\mathrm{Au}$ & \\
Thermal conductivity & 0.105 & $\mathrm{~W} \mathrm{~mm}-1 \mathrm{~K}^{-1}$ \\
Thermal expansion coefficient & $3.5 \times 10^{-6}$ & $\mathrm{~K}^{-1}$ \\
Young's modulus & $2.41 \times 10^{6}$ & $\mathrm{~N} \mathrm{~mm}$ \\
Poisson ratio & 0.3 & \\
Film coefficient & $7.5 \times 10^{-4}$ & $\mathrm{~W} \mathrm{~mm}$ \\
\hline
\end{tabular}

Table 4. Dimensions of the mirrors and radiation spot sizes.

\begin{tabular}{lll}
\hline Parameter & Value & Units \\
\hline Mirror 1: blank size $(\mathrm{L} \times \mathrm{W} \times \mathrm{T})$ & $400 \times 80 \times 50$ & $\mathrm{~mm}^{3}$ \\
Mirror 1: reflecting area $(\mathrm{L} \times \mathrm{W})$ & $400 \times 6$ & $\mathrm{~mm}^{2}$ \\
Mirror 1: spot size & $\pi \times 200.00 \times 2.43$ & $\mathrm{~mm}^{2}$ \\
Mirror 2: blank size $(\mathrm{L} \times \mathrm{W} \times \mathrm{T})$ & $350 \times 50 \times 30$ & $\mathrm{~mm}^{3}$ \\
Mirror 2: reflecting area $(\mathrm{L} \times \mathrm{W}) \dagger$ & $350 \times 15$ & $\mathrm{~mm}^{2}$ \\
Mirror 2: spot size at $250 \mathrm{eV}$ & $\pi \times 1.74 \times 14.54$ & $\mathrm{~mm}^{2}$ \\
Mirror 2: spot size at $2 \mathrm{keV}$ & $\pi \times 1.74 \times 78.59$ & $\mathrm{~mm}^{2}$ \\
\hline
\end{tabular}

$\lceil$ Since the spot size overcomes the mirror edge the effective absorbed power decreases. This effect is taken into account in the structural analysis. 
Table 5. Parameters of the monochromator in the cases considered.

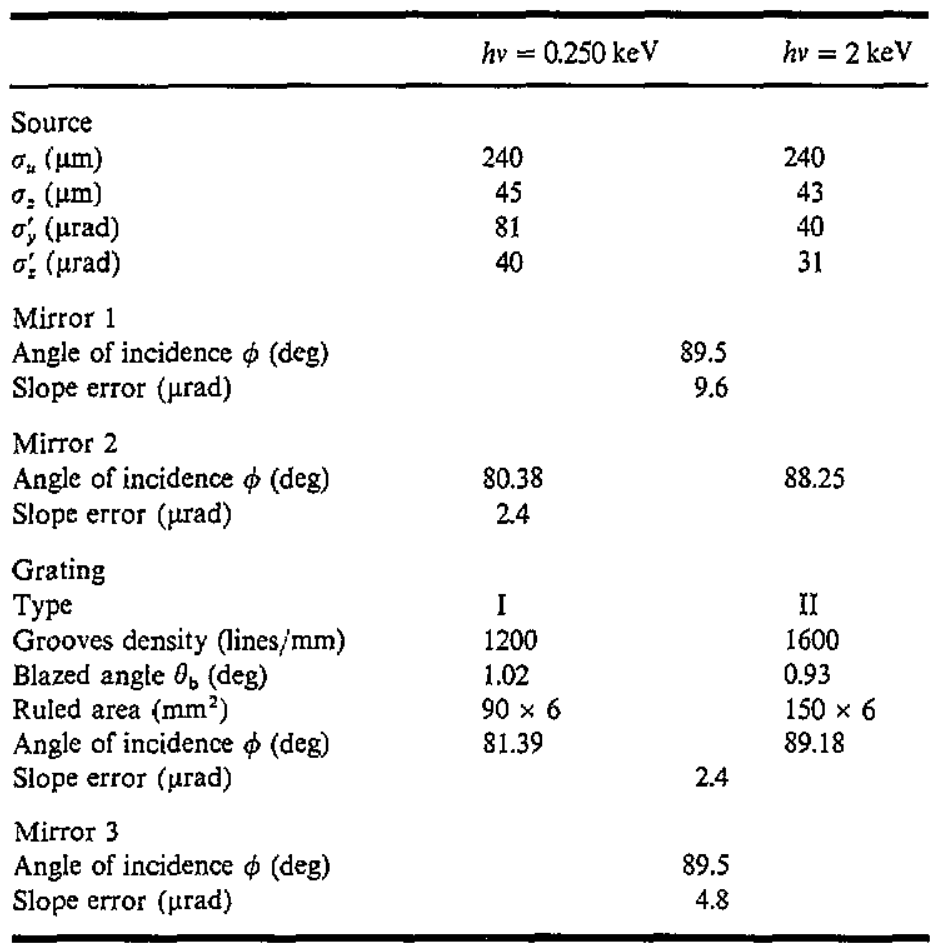

cooling scheme. The plane mirror, instead, receives higher thermal loads that are not symmetrically distributed on the surface. At $250 \mathrm{eV}$ the grazing angle is $9.62^{\circ}$ and the beam hits the surface near the remote edge of the mirror, while at $2 \mathrm{keV}$ the angle is $1.7^{\circ}$ and the spot lies near the first edge. The spot sizes, reported in table 5, are different in the two cases as well as the absorbed power distributions. These asymmetries imply an increment of the bending of the surface since the heat flows only along one side of the optical surface and the corresponding mechanical stress is not uniform. The blank is cooled only on the backside. Since the spot size is smaller than the blank in both directions, the calculated displacements in the intersection node are slightly different. We have considered the average values and renormalized all the displacements. Even if the absolute temperatures are not relevant, around $40^{\circ} \mathrm{C}$ with respect to a coolant bulk temperature of $20^{\circ} \mathrm{C}$, the resulting deformations are not negligible. The surface grows up to $4 \mu \mathrm{m}$ at $250 \mathrm{eV}$ and $2.1 \mu \mathrm{m}$ in the high energy range (see figure 3 ). The corresponding thermal slope reaches the maximum at the edge of the impinging beam area. Along the longitudinal direction, it amounts to $\approx 10$ seconds of arc for the low energy case and $\approx 4$ seconds of arc in the other case, as figure 4 illustrates. In the sagittal direction the surface grows more uniformly and the slope reaches $\approx 4$ seconds of arc for the first case and $\approx 2$ seconds of arc in the second.

In conclusion, differences in the amount of slope in the two directions is an obvious consequence of the elongated shape of the spot size in comparison with the total mirror surface and especially of the eccentricity of impinging beam with respect to the centre of the surface. These structural results have been used in the ray tracing simulation for 


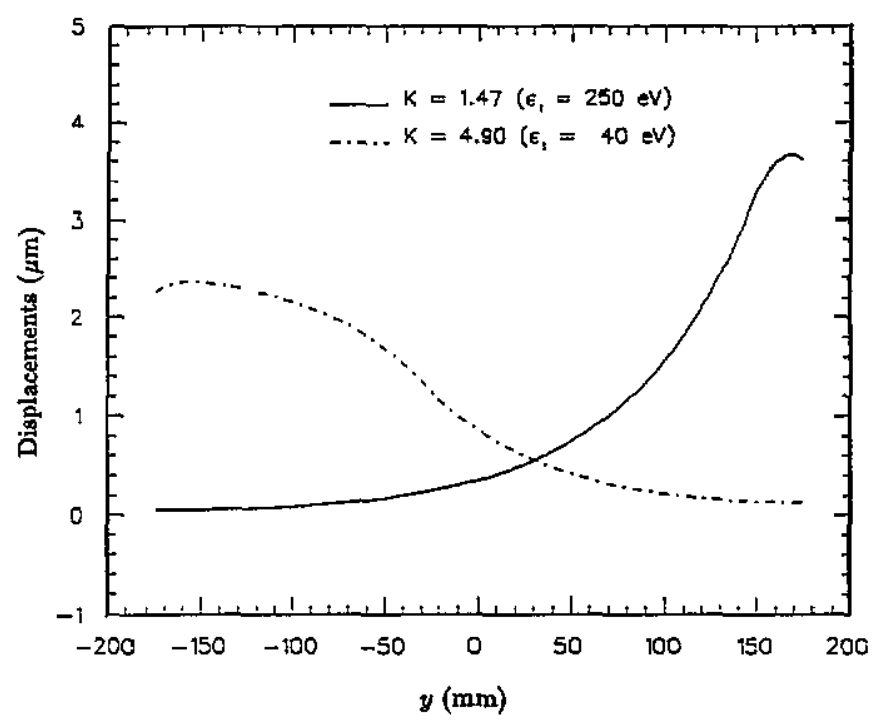

Figure 3. Surface displacements on the central longitudinal cross section for mirror 2 . The full curve corresponds to the deformations induced by low energy photons $(K=1.47)$, the broken curve to high energy photons $(K=4.90)$.

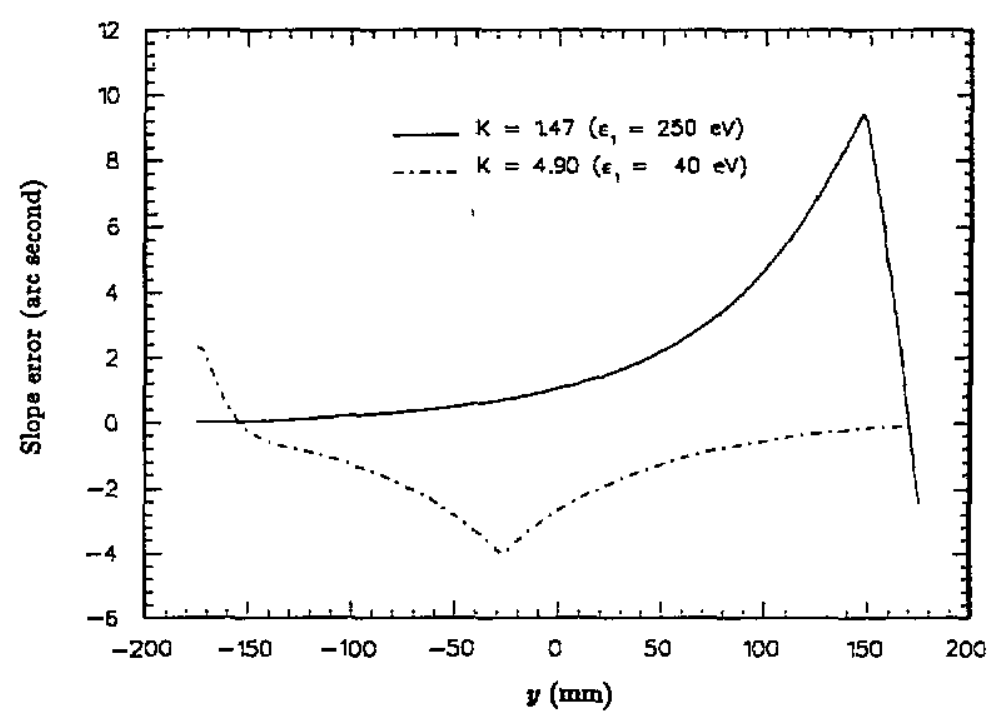

Figure 4. Surface thermal slope errors on the central longitudinal cross section for mirror 2. The full curve corresponds to the slope induced by low energy photons $(K=1.47)$, the broken curve to high energy photons $(K=4.90)$.

obtaining quantitative evaluation of the thermal effects on the performance of the optical instrument.

\section{Manufacturing slope errors}

The most difficult aspects of optical manufacturing of $\mathrm{x}$-ray quality mirrors are slope error reduction and surface roughness. The latter cannot be included in a convenient 
form in a ray tracing program as the one used here. It is well known (see e.g. [8]), however, that severe reduction in the efficiency of reflection occurs at short wavelengths due to this effect and the net result on the focal plane of an optical system is the appearance of a uniformly illuminated background, with a loss in intensity contrast and spatial resolution. Surface roughness is dependent upon material and shape of the optical surface considered. Quantitatively, the best values obtained so far range from 1 to $2 \AA$ RMS for plane or spherical surfaces and from 4 to $5 \AA$ RMS for aspherics.

While surface roughness is related to short range imperfections of the optical surfaces in the $x$-ray wavelength scale, slope errors are related to the long range imperfections of a reflecting surface, that is to the deviations from nominal figure of a surface. They are determined by the compound errors in the figuring of the surface and are dependent upon material and final figuring and polishing processes. Values range from fraction of arc seconds for plane to several arc seconds for aspherical surfaces, as can be seen in table 5 .

The simulation of slope errors due to the tolerances in fabrication may be performed by imposing appropriate deviations to the reflected/diffracted ray. In fact, a change of an angle $\delta$ in the slope of the surface corresponds to a similar deviation of the normal at the same location. Therefore, one may reproduce the effect simply by rotating by an appropriate amount the vector representing the perpendicular to the optical surface and then performing the calculations of the reflected/diffracted ray as in the usual case. The deviation angle may follow appropriate statistical distributions. In our case, a Gaussian distribution for the absolute deviation $\theta$ from the geometrical perpendicular has been chosen with random orientations $\varphi$ around the azimuthal direction. This choice is justified by the lack of more precise information on the actual slope error distribution. In the future, when a specific optical mirror will be selected, experimental information obtained with, e.g. high resolution profilometry could be used to improve the modelling, as has recently been reported [9].

\section{Ray tracing code}

A ray tracing code has been used to evaluate the resulting ray pattern at the focal plane of the monochromator, taking into account slope errors and thermal load effects. Rays originating at the source are followed through the optical path using reflection and diffraction laws at each mirror or grating. The positions of the source, of each optical surface and of the focal plane are determined by orthogonal reference frames centred at each vertex with the $x$-axis normal to the surface.

In order to simulate the beamline intensity pattern, the source of rays is made up with several point sources located on the source plane according to a Gaussian distribution along the tangential and sagittal directions. From each point source a number of rays depart distributed in directions according to Gaussians with $1 / \mathrm{e}$ halfwidths in the two orthogonal directions matching beamline specifications. For the simulations presented below, we have used sources consisting of up to 900 rays.

Our optical calculations take into account the thermal induced slope errors for the plane mirror only, since, as discussed in the previous sections, substantial heating and deformation occurs only for that element.

For each optical configuration, two cross sections of the mirror along the symmetry axes of the elliptical heating pattern were considered and deformations evaluated with the two-dimensional thermal code. In order to obtain a three-dimensional displacement 
distribution over the whole mirror surface, we have normalized each transverse distribution to the value of the longitudinal distribution at the crossing point. The displacements of the optical surface are interpolated by two-dimensional cubic splines. The coefficients together with first derivatives of the displacements in sagittal and tangential directions are then used by the ray tracing program. For each ray impinging on the surface, a new impact point and normal to the surface is calculated before evaluating the direction of the reflected ray. If required, further modifications to the normal at the impact point are added to simulate the surface slope errors, as explained in section 4. Once the resulting ray pattern at the focal plane of the system is obtained, the coordinates of the centre as well as the standard deviations are evaluated in the dispersion direction (vertical) and parallel to the exit slit. Also the number of transmitted rays is evaluated which gives a measure of the geometrical throughput of the system.

\section{Results at $250 \mathrm{eV}$ and $2 \mathrm{keV}$}

The set of figures 5-8 illustrate the results obtained at $h v=250 \mathrm{eV}$. The configurations for both ALOISA and the spectrometer which have been considered are summarized in tables 1 and 5, respectively. Table 6 summarizes the results obtained, i.e. the position of the centre of the focal image, standard deviations and the fraction $T$ of input rays reaching the focal plane. The ray source for the simulations is the one illustrated in the previous section. Note that all ray pattern plots have the same scale lengths, the centring

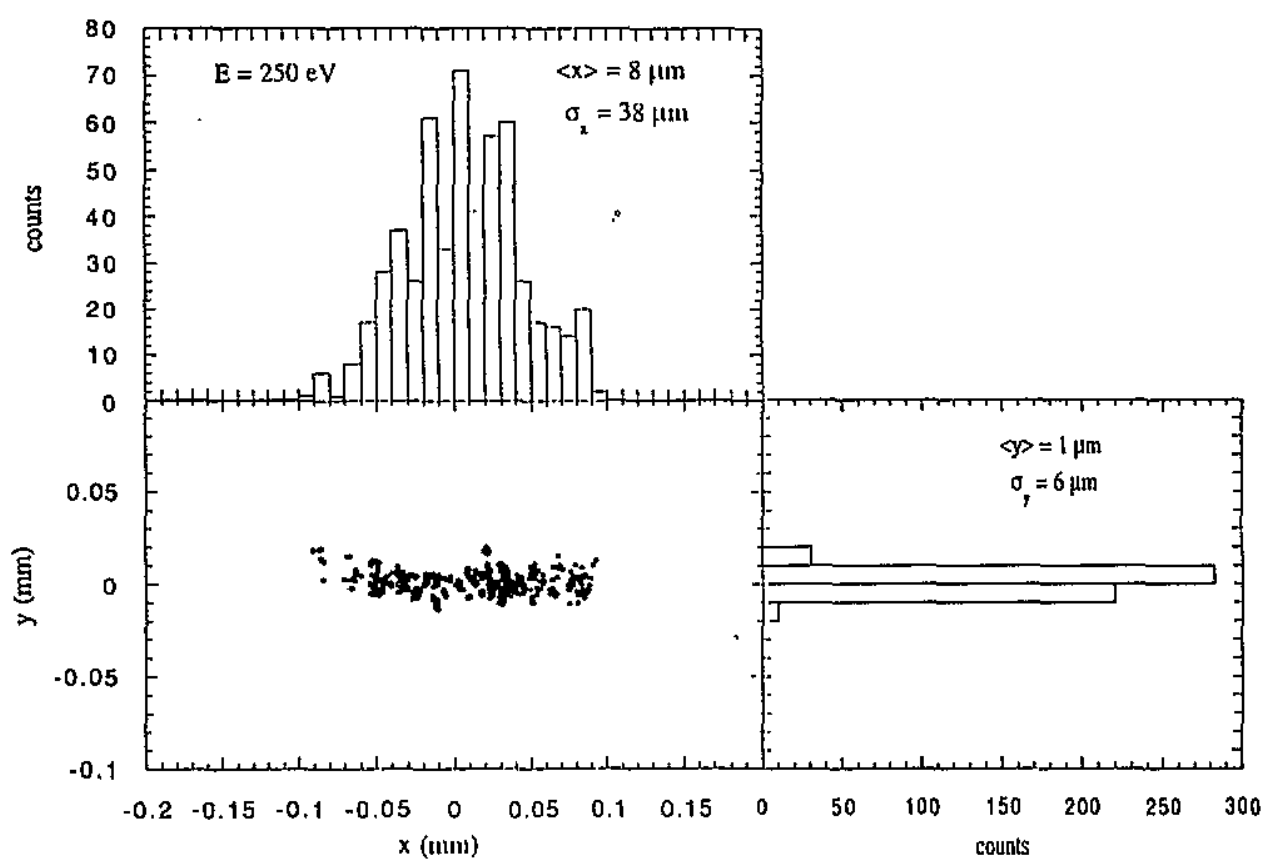

Figure 5. Simulated focal spot image and corresponding histograms along (vertical) and across (horizontal) dispersion direction at $250 \mathrm{eV}$ by an extended source matching ALOISA specifications (see table 6). Nominally perfect optical surfaces and no thermal deformations are assumed. 
Table 6. Positions $\langle x\rangle,\langle y\rangle$, standard deviations $\sigma_{x}, \sigma_{y}$ of the focal spots and transmission $T$ in different configurations of the monochromator. ES, extended source; $\mathrm{TL}$, thermal load; SE, slope errors.

\begin{tabular}{llllrlrl}
\hline & $\begin{array}{l}h v \\
(\mathrm{keV})\end{array}$ & $\begin{array}{l}\lambda \\
(\AA)\end{array}$ & $\begin{array}{l}\langle x\rangle \\
(\mu \mathrm{m})\end{array}$ & $\begin{array}{l}\langle y\rangle \\
(\mu \mathrm{m})\end{array}$ & $\begin{array}{l}\sigma_{x} \\
(\mu \mathrm{m})\end{array}$ & $\begin{array}{l}\sigma_{y} \\
(\mu \mathrm{m})\end{array}$ & $\begin{array}{l}T \\
(\%)\end{array}$ \\
\hline $\mathrm{ES}$ & 0.25 & 49.6 & 8 & 1 & 38 & 6 & 60 \\
$\mathrm{ES}+\mathrm{TL}$ & 0.25 & 49.6 & 4 & -197 & 35 & 19 & 58 \\
$\mathrm{ES}+\mathrm{SE}$ & 0.25 & 49.6 & -2 & 0 & 56 & 14 & 59 \\
$\mathrm{ES}+\mathrm{TL}+\mathrm{SE}$ & 0.25 & 49.6 & 6 & -197 & 55 & 22 & 59 \\
$\mathrm{ES}$ & 2 & 6.2 & 0 & 0 & 30 & 3 & 79 \\
$\mathrm{ES}+\mathrm{TL}$ & 2 & 6.2 & -1 & 6 & 37 & 5 & 81 \\
$\mathrm{ES}+\mathrm{SE}$ & 2 & 6.2 & 7 & 0 & 56 & 8 & 82 \\
$\mathrm{ES}+\mathrm{TL}+\mathrm{SE}$ & 2 & 6.2 & 6 & 5 & 57 & 9 & 80 \\
\hline
\end{tabular}

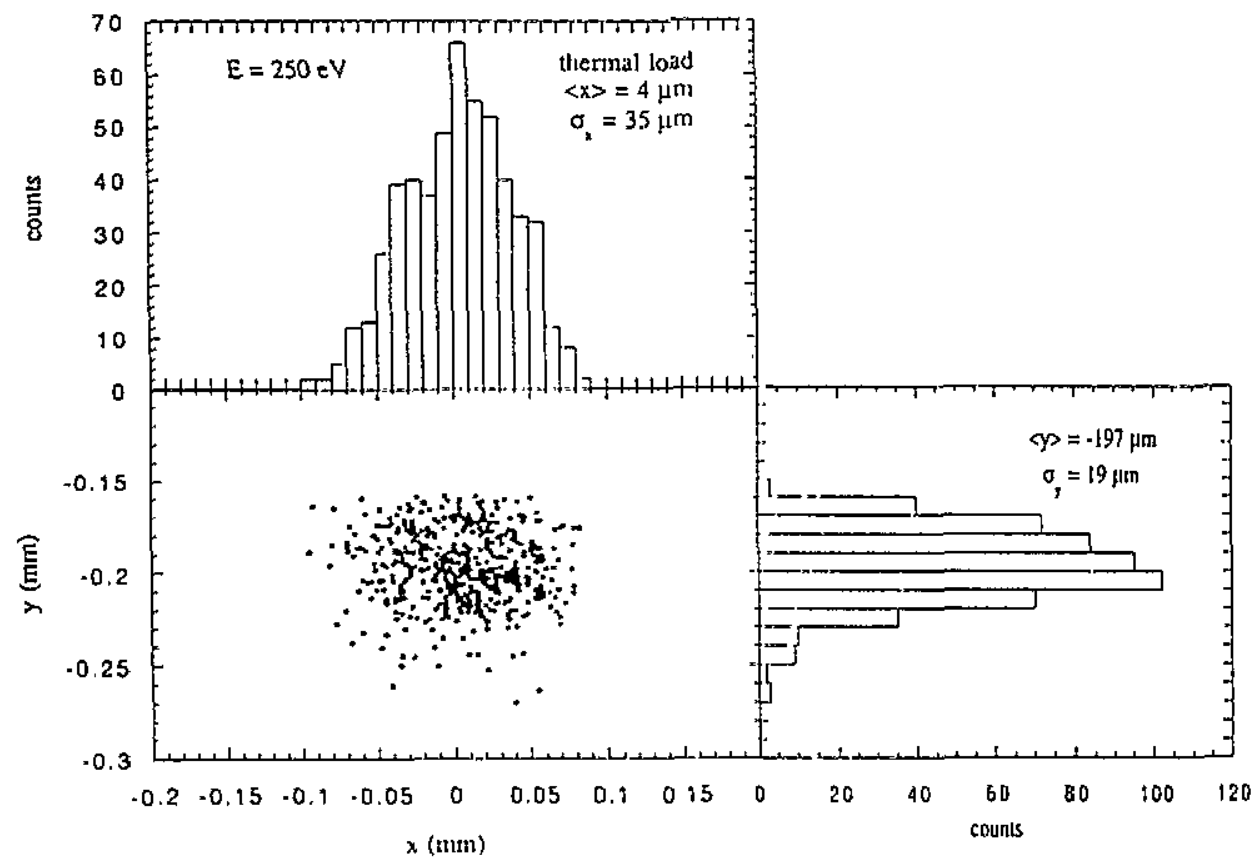

Figure 6. As in figure 5 but introducing the thermal load on mirror 2 , as discussed in the text.

being in some cases different. This result refers to the lowest energy selection for the monochromator.

Several runs were made in different conditions so as to observe separately the various effects. Figure 5 depicts the focal plane pattern originated by the broad source alone with nominally perfect optics and alignment. The pure aberrations due to the finite source size result here in a broadening of the spot with standard deviations of $6 \mu \mathrm{m}$ in the dispersion direction and of $38 \mu \mathrm{m}$ in the horizontal direction. The shape of the spot shows that the conditions are optimized for focusing in the dispersion direction. These 


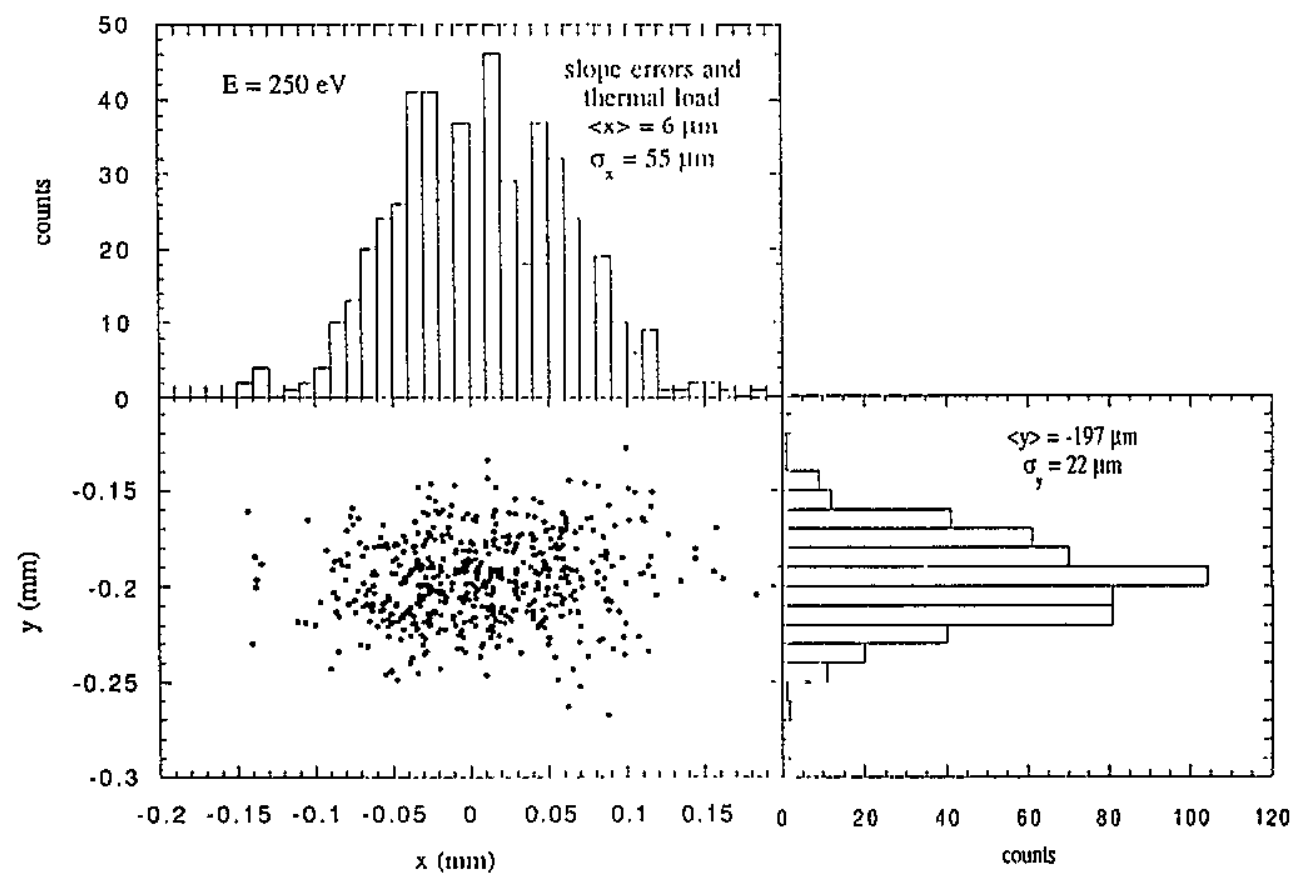

Figure 7. As in figure 5 but inserting the slope errors of table 5 for each optical surface, as discussed in the text.

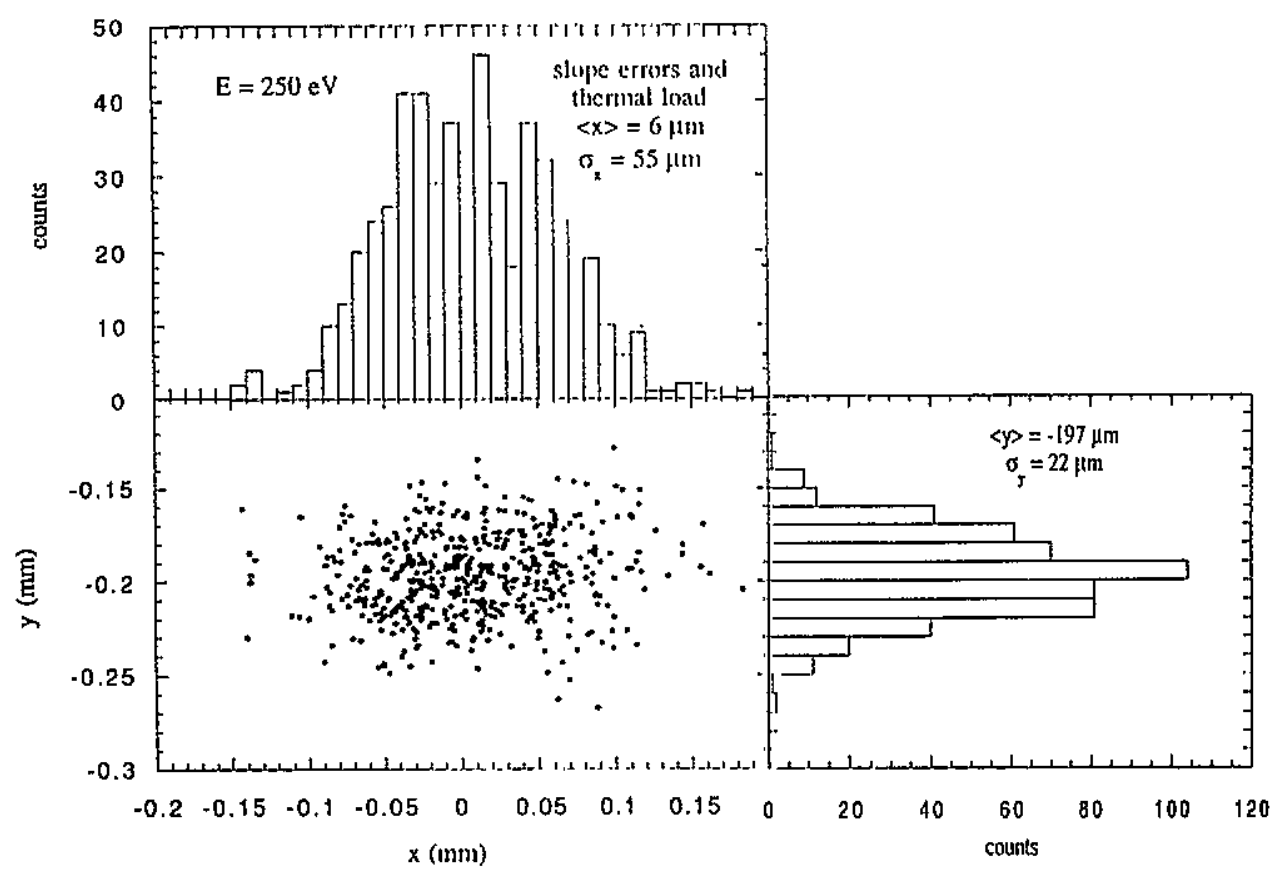

Figure 8. Simulation of the focal spot at $250 \mathrm{eV}$ from an extended source including both thermal deformations of mirror 2 and slope errors for each optical surface. 
results, however, degrade considerably as soon as slope errors are introduced. The variances of the slope error distributions for each surface are those given by manufacturers of XUV mirrors and are listed in table 5 with the other instrument parameters. Slope errors contribute to spot broadening with a standard deviation $\sigma \approx 14 \mu \mathrm{m}$ in the dispersion direction, as seen in figure 7. This value is to be compared with a standard deviation $\sigma \approx 19 \mu \mathrm{m}$ obtained in the case of pure thermal load on mirror 2 (figure 6). The compound effect is illustrated in figure 8 . In the dispersion direction the resulting spot has a standard deviation of $22 \mu \mathrm{m}$, corresponding to a spectral broadening of $\approx 0.01 \AA$. In the horizontal direction, parallel to the slit jaws, we obtain a standard deviation $\sigma \approx 55 \mu \mathrm{m}$. The thermal load on mirror 2 does not substantially affect the overall performances of the instrument.

The major effect attributable to thermal loads, however, is a vertical downward shift of the image of $-197 \mu \mathrm{m}$. An explanation of this effect is the following: since the heating of mirror 2 occurs at the edge of the mirror itself, the resulting deformation is more pronounced near the edge of the mirror. This asymmetry decreases the angle of incidence of the rays. The rays impinge then onto the grating at a slightly smaller angle than the nominal value. The result is that the direction of the rays onto the paraboloid is not horizontal but is slightly tilted downward, resulting in a lower position of the focal image. The wavelength component which is centred onto the exit slit of the monochromator is in this case shifted by $\approx+0.1 \AA$ with respect to the nominal value. Its focal pattern does not appear broadened with respect to the nominal focused image. Simulations have resulted in standard deviations of 58 and $21 \mu \mathrm{m}$ in the horizontal and vertical direction, respectively. It is clear, however, that appropriate wavelength calibrations at different intensity levels of the input radiation will be necessary during operation.

The results of the simulations at $2 \mathrm{keV}$ are illustrated in figures $9-12$. The parameters of the spectrometer which have been changed are the grating, now $1600 \mathrm{lines} / \mathrm{mm}$ and with a $0.93^{\circ}$ blaze angle, and the angles of incidence of the beam on mirror 2 and grating. These are listed in table 5. The focal spot sizes found reflect the smaller thermal load on mirror 2 suffered in this configuration which is determined by the larger angles of incidence used at these photon energies. The broadening of the focal spot due to thermal effects is quite weak, its inclusion corresponding to an increase from 8 to $9 \mu \mathrm{m}$ of the standard deviation in the dispersion direction. The lower absolute values of the focal spot sizes are due mainly to the better collimation of the monochromatic source beam in this spectral region, as shown in table 5. Moreover, the illuminated area on the plane mirror is more spread out in the longitudinal direction so that the slopes due to thermal gradients are less pronounced. This determines a smaller deviation of the centre-ofgravity of the beam. On the focal plane, this results in a shift of less than $+5 \mu \mathrm{m}$, in contrast with the $\approx-200 \mu \mathrm{m}$ of previous case. The change of sign in the position is directly related to the asymmetry of the deformation of the reflecting surface of mirror 2 in the two cases.

\section{Conclusions}

The first step of the present study has been to evaluate quantitatively the absorbed power on each optical element of the monochromator. Substantial heating has been observed for the first two mirrors. Holders for the optics equipped with cooling channels, are therefore required for these elements. A similar solution has been proposed in a 


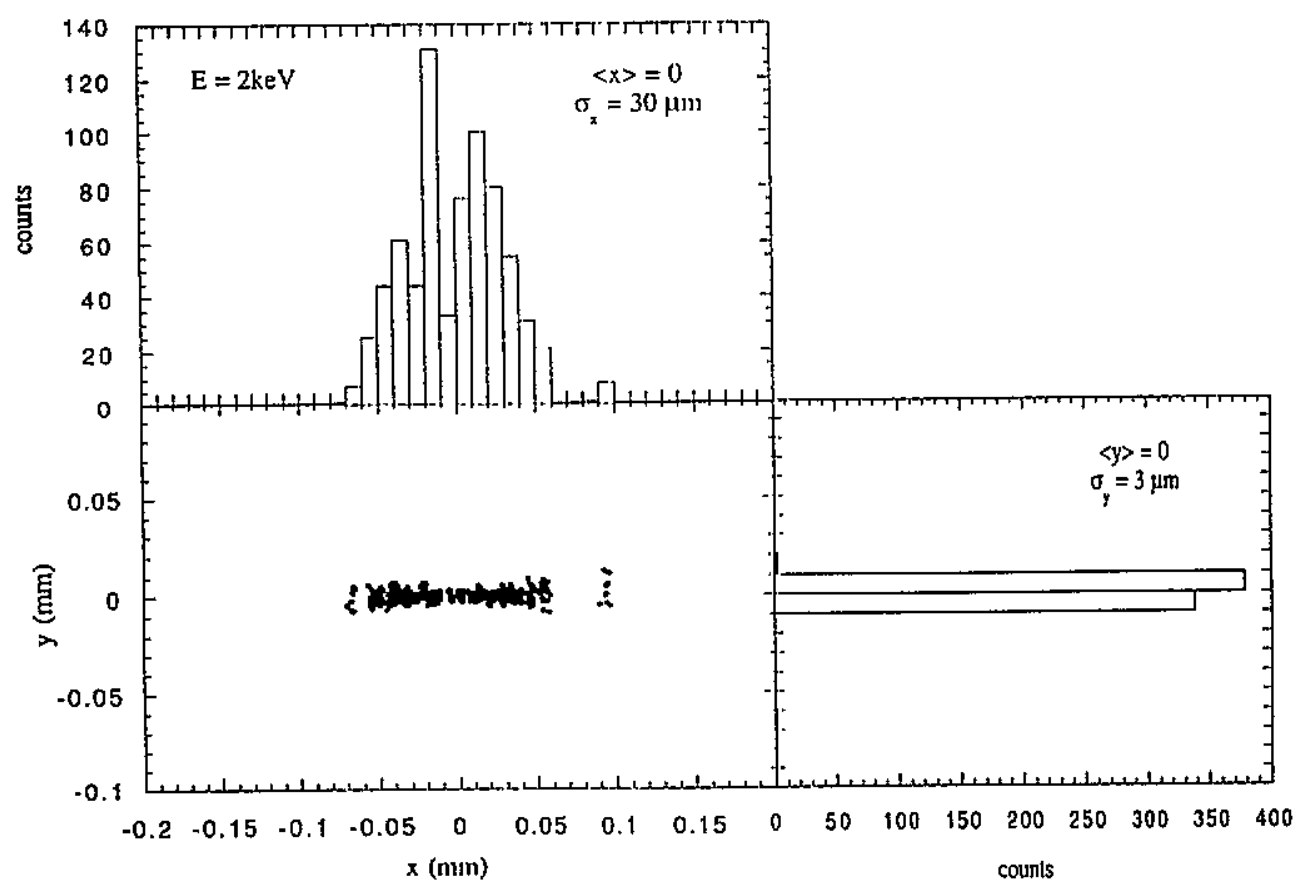

Figure 9. Simulated focal spot image and corresponding histograms along (vertical) and across (horizontal) dispersion direction at $2 \mathrm{keV}$ by an extended source matching ALoISA specifications (see table 5). Nominally perfect optical surfaces and no thermal deformations are assumed.

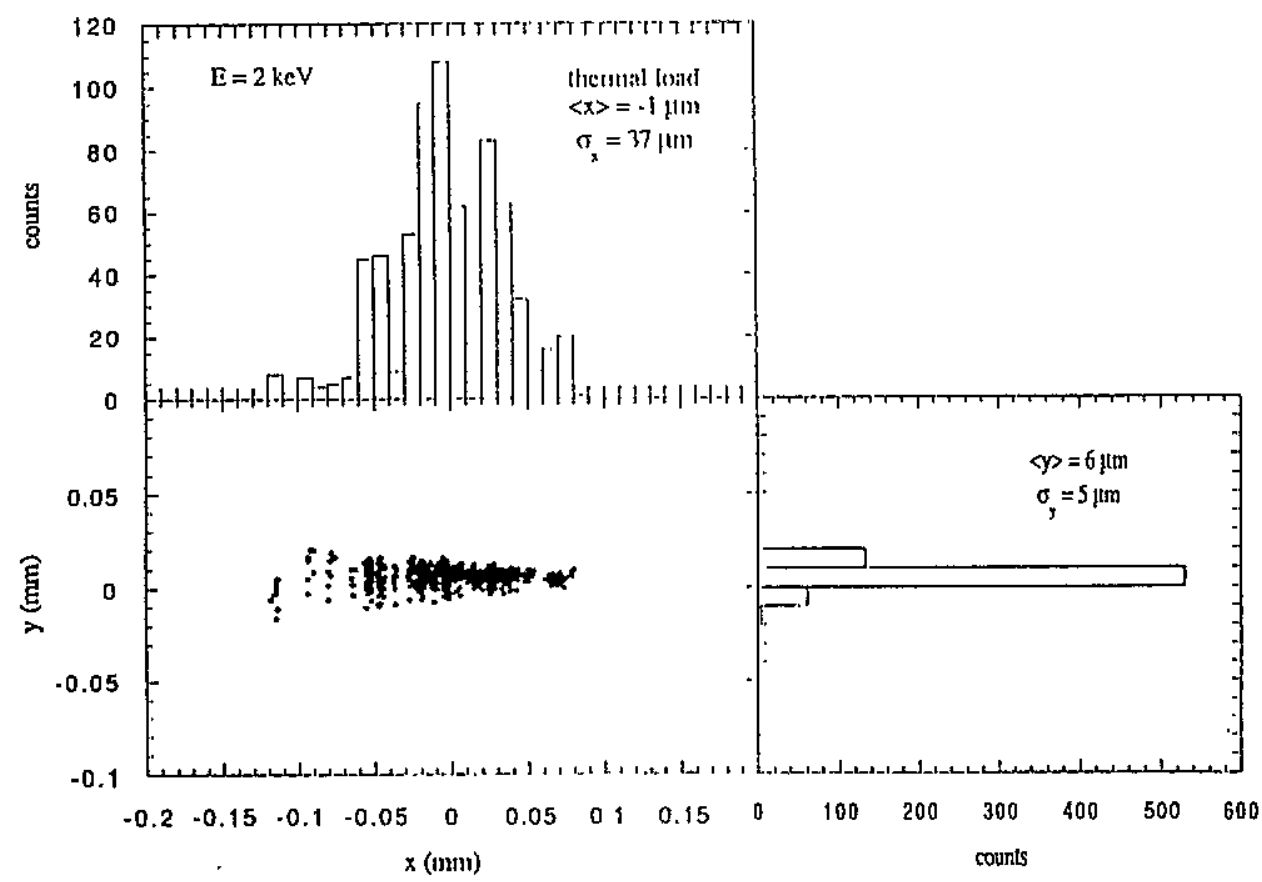

Figure 10. As in figure 9 but introducing the thermal load on mirror 2 , as discussed in the text. 


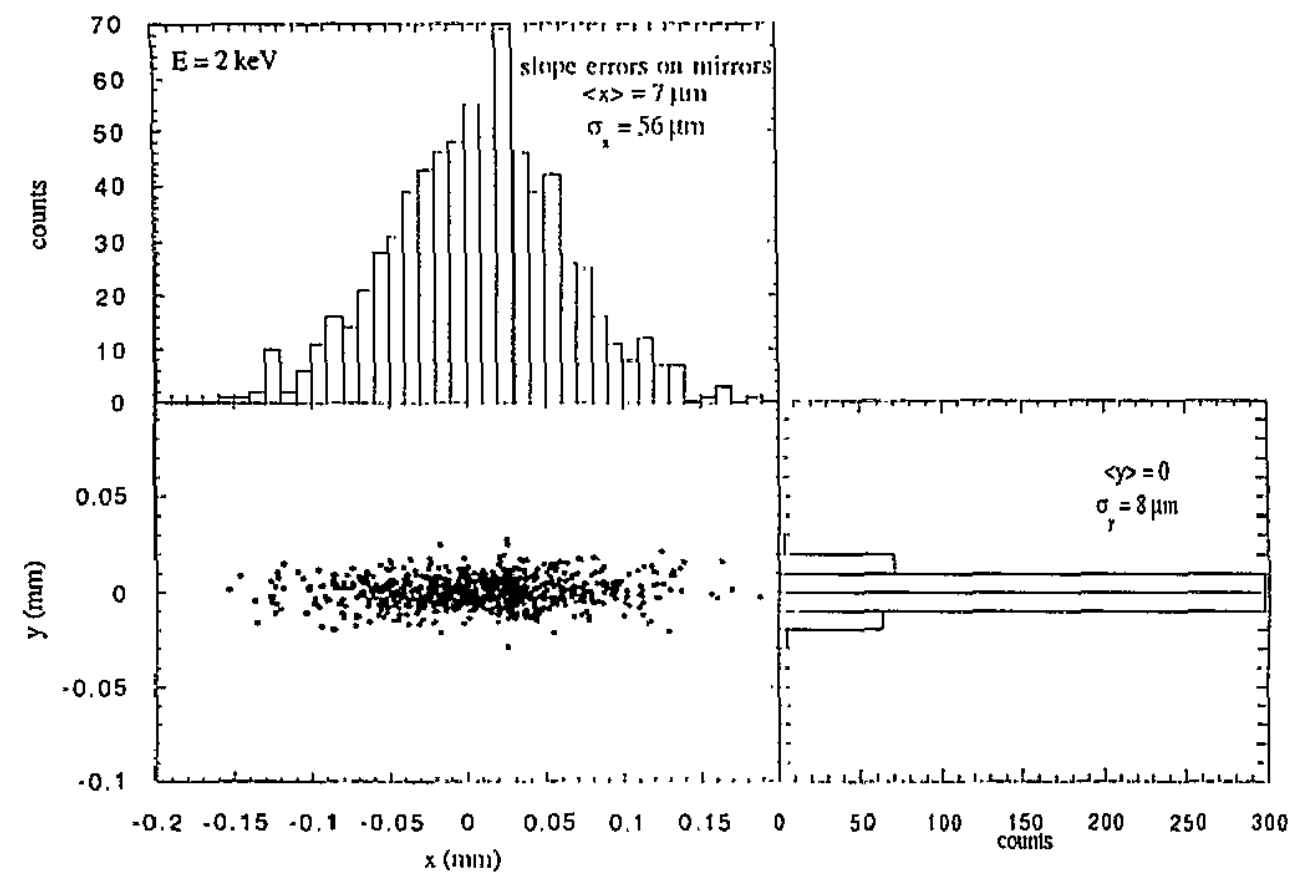

Figure 11. As in figure 9 but inserting the slope errors of table 5 for each optical surface, as discussed in the text.

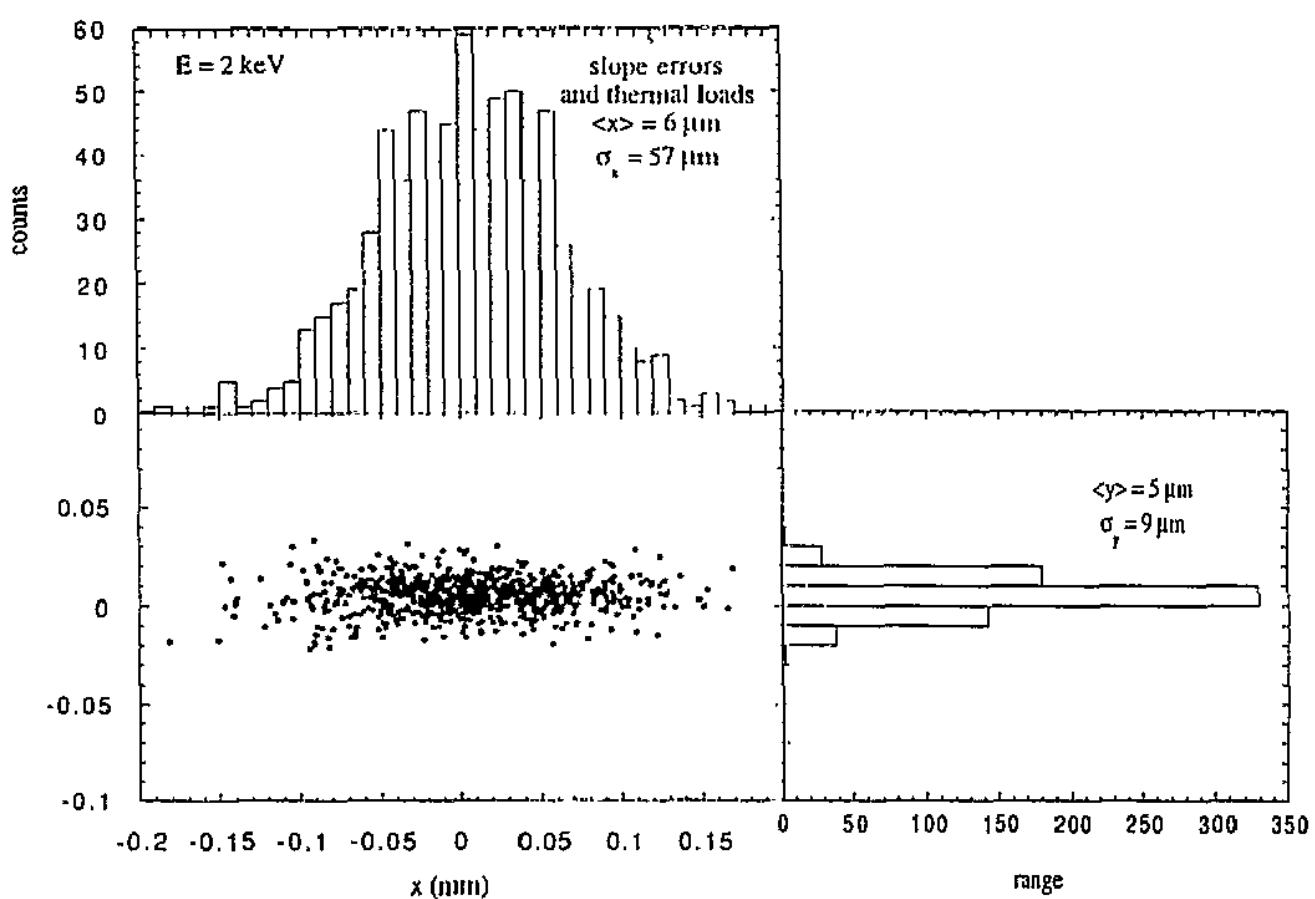

Figure 12. Simulation of the focal spot at $2 \mathrm{keV}$ from an extended source including both thermal deformations of mirror 2 and slope errors for each optical surface. 
parallel study $[10,11]$, for the first diffraction crystal in the high energy dispersive system. Such conventional cooling geometries appear well suited for the present application, even in the case of greater thermal loads imposed by a beam generated from, i.e. extra undulator sections inserted in the ring.

A detailed analysis carried out with ray tracing has elucidated the role of both thermal induced deformations and manufacturing slope errors on the optical performances. With the inclusion of all the detrimental effects, a limited broadening of the focal spot in the whole energy range has been observed. Standard deviations in the direction of the slit jaws amounting to $\approx 55 \mu \mathrm{m}$ have been calculated at the extremes of the spectral range. In the dispersion direction, instead, the broadening ranges from $9 \mu \mathrm{m}$ at $2 \mathrm{keV}$ to $22 \mu \mathrm{m}$ at $250 \mathrm{eV}$.

The asymmetry of the heating on the plane mirror is the major cause of a spectral shift of the radiation selected by the exit slit. It may be as large as $0.1 \AA$ at $250 \mathrm{eV}$.

The contribution to the total spectral broadening of slope errors is comparable to those due to heating of the optical elements. Unfortunately, the thermal slopes appear to be largely independent of the cooling geometry, and cannot be substantially reduced. However, better performances of the instrument in this spectral region may still be achieved by improving the quality of the optical elements only, i.e. with the same thermal deformations.

Additionally, we have investigated whether the superposition of different degrading effects does not offset the advantages of sagittal focusing performed by the second paraboloid. This is motivated by the observation that a tangential configuration considerably reduces the spectral broadening due to thermal deformations only. The results show that this is not the case. The choice of sagittal focusing appears still the most favourable even in the presence of thermal loads.

The calculations have therefore shown that spatial and spectral resolutions are mainly limited by the optical manufacturing state-of-the-art. Progress in optical performances are expected by improved figuring of the optical elements.

\section{Acknowledgments}

We are indebted to R Baruzzo, G Naletto and $G$ Tondello for useful comments and discussions.

\section{References}

[1] Naletto G and Tondello G 1992 Pure Appl. Opt. 1347

[2] Bonfante $G$ and Tondello G 1990 Appl. Opt. 294250

[3] Walker R P 1990 Internal note

[4] Walker R P 1991 Internal note

[5] Walker R P and Diviacco B 1992 Rev. Sci. Instrum 63392

[6] Henke B L, Lee P, Tanaka T J, Shimabukuro R L and Fujikawa B K 1982 At. Data Nucl. Data Tables 271

[7] ABAQUS-Rel. $4.91991 \mathrm{HKS}$ Inc

[8] Takacs P Z and Church E L 1990 Nucl. Instrum. Methods A 291253

[9] ESRF Users' Information Meeting (Grenoble) 1991 papers by C Riekel, P Boseke and S Ferrer

[10] Lenardi C 1993 Nuovo Cimento in press

[11] Bernstorff S and Busetto E 1992 Internal Report ST/S-R-92/22, Sinchrotrone Triese

[12] Iazzourene F 1991 Trieste Synchrotron Radiation Facility ELETrRA-Parameter List 